ARTICLE

DOI: $10.1038 / \mathrm{s} 41467-018-06146-9$

\title{
Somatic Trp53 mutations differentially drive breast cancer and evolution of metastases
}

Yun Zhang ${ }^{1,4}$, Shunbin Xiong ${ }^{1}$, Bin Liu1, Vinod Pant ${ }^{1}$, Francis Celii ${ }^{1}$, Gilda Chau ${ }^{1}$, Ana C. Elizondo-Fraire ${ }^{1}$ Peirong Yang ${ }^{1}$, Mingjian James You ${ }^{2}$, Adel K. El-Naggar ${ }^{3}$, Nicholas E. Navin ${ }^{1} \&$ Guillermina Lozano (D) ${ }^{1}$

TP53 mutations are the most frequent genetic alterations in breast cancer and are associated with more aggressive disease and worse overall survival. We have created two conditional mutant Trp53 alleles in the mouse that allow expression of Trp53R172H or Trp53R245W missense mutations in single cells surrounded by a normal stroma and immune system. Mice with Trp53 mutations in a few breast epithelial cells develop breast cancers with high similarity to human breast cancer including triple negative. p53R245W tumors are the most aggressive and exhibit metastases to lung and liver. Development of p53R172H breast tumors with some metastases requires additional hits. Sequencing of primary tumors and metastases shows p53R245W drives a parallel evolutionary pattern of metastases. These in vivo models most closely simulate the genesis of human breast cancer and will thus be invaluable in testing novel therapeutic options.

\footnotetext{
${ }^{1}$ Department of Genetics, University of Texas MD Anderson Cancer Center, Houston, TX 77030, USA. ${ }^{2}$ Department of Hematopathology, University of Texas MD Anderson Cancer Center, Houston, TX 77030, USA. ${ }^{3}$ Department of Pathology, University of Texas MD Anderson Cancer Center, Houston, TX 77030, USA. ${ }^{4}$ Present address: Department of Pharmaceutical and Environmental Health Sciences, College of Pharmacy, Texas Southern University, 
B reast cancer is a heterogeneous disease generally comprised of five molecular subtypes: luminal A, luminal B, normallike, HER-2 enriched, and basal-like ${ }^{1,2}$, with the last two subtypes having poorer prognosis and the highest TP53 mutation frequency ${ }^{2}$. In particular, the majority of these mutations occur on three arginines, R175, R248, and R273, in the DNA binding domain of TP53, with R248 being the top mutational hotspot in breast cancer ${ }^{2}$. To investigate the role of $\operatorname{Trp} 53$ mutations in breast cancer, a number of mouse models have been established. For example, mice containing germline Trp53 mutations model Li-Fraumeni Syndrome (LFS) ${ }^{3}$, an inherited cancer syndrome associated with germline Trp53 mutations, and develop highly metastatic mammary tumors in a Balb/C background. On the other hand, TP53 mutations are largely spontaneous and somatic ${ }^{4}$. The heretofore published $\operatorname{Trp} 53$ alleles, which allow spontaneous expression of mutant Trp53, have indeed played an important part in advancing our understanding of somatic p53 function in breast cancer, but exhibit critical caveats that undermine their clinical faithfulness. For example, a number of transgenic lines, which overexpress mutant Trp53 in mouse mammary glands, have been particularly useful for understanding the effect of different forms of mutant Trp53 in mammary development and tumorigenesis, as mutant $\operatorname{Trp} 53$ transgenes are often driven by mammary epithelium specific promoters ${ }^{5,6}$. However, such models usually rely on random integration of multiple copies of Trp53, which are present together with two endogenous WT Trp53 alleles, and therefore the expression levels and activities of the Trp53 transgene may not accurately model endogenous mutated TP53 alleles observed in human tumors. In addition, two knock-in conditional $\operatorname{Trp} 53 \mathrm{R} 172 \mathrm{H}$ (corresponding to human TP53R175H) and Trp53R270H (corresponding to human TP53R273H) alleles (referred to as $\operatorname{Trp} 53^{L S L-m u t}$ ), in which a loxP flanked STOP cassette was inserted into the first intron to block mutant Trp53 expression, have been utilized to study different types of cancers including breast cancer ${ }^{7-9}$. However, it is important to note that insertion of the STOP cassette makes the whole Trp53 $3^{L S L-m u t /}+$ mouse heterozygous for $\operatorname{Trp53}$, which complicates the interpretation of the tumor phenotypes since studies have shown that impaired $p 53$ status in tumor microenvironment facilitates tumor development ${ }^{10,11}$.

Therefore, better models that faithfully mimic human sporadic breast cancer involving somatic TP53 mutations are needed. These models will be critical for determining if and how expression of spontaneous p53 mutant alone in a limited number of epithelial cells can drive breast tumorigenesis, whether different hot spot $p 53$ mutations have the same potency, and whether these breast tumors could, at least partially, recapitulate characteristics of spontaneous human breast cancers. In addition, the emerging evidence for inter-patient heterogeneity and the notion of personalized therapy urge an understanding of the breast cancers specifically driven by $p 53$ mutations. It will be important to understand the heterogeneity and mutational evolution of breast tumors initiated by somatic $p 53$ mutations, which may enable tailoring of therapeutic strategies to the fundamental molecular lesions driving a particular tumor.

In this study, we generate two conditional Trp53 alleles, which allow us to focally convert wild type (WT) p53 into a mutant, $\mathrm{p} 53 \mathrm{R} 172 \mathrm{H}$ or p53R245W (corresponding to human $\mathrm{p} 53 \mathrm{R} 175 \mathrm{H}$ and p53R248W, respectively), in mammary epithelial cells while maintaining WT p53 in the rest of the mouse. We find that p53R245W is more potent in driving mammary tumorigenesis and metastasis than $\mathrm{p} 53 \mathrm{R} 172 \mathrm{H}$. In addition, multi-region whole exome sequencing reveals inter- and intra-tumor heterogeneity, as well as a parallel mutational evolutionary pattern in p53R245W-driven primary tumors and associated metastases.

\section{Results}

Generation of the $\operatorname{Trp53} 3^{w m-R 172 H}$ and $\operatorname{Trp5} 3^{w m-R 245 W}$ alleles. To study how a somatic $p 53$ missense mutation drives breast cancer development, we generated two conditional Trp53 alleles, which allowed us to convert WT $\operatorname{Trp} 53$ to either $\operatorname{Tr} p 53 \mathrm{R} 172 \mathrm{H}$ or $\operatorname{Trp} 53 R 245 \mathrm{~W}$ and to generate a $\operatorname{Trp} 53$ missense mutation in a single cell or tissue in vivo. Briefly, a partial WT Trp53 cDNA encoding exons $5-11$ or exons 7-11 flanked by loxP sites was inserted into intron 4 or 6 of the endogenous locus of $\operatorname{Trp53}$ carrying a specific mutation in exon 5 or 7 , respectively (Fig. 1a and Supplementary Fig. 1a, b). These alleles named $\operatorname{Trp} 53^{w m-R 172 H}$ or $\operatorname{Tr} p 53^{w m-R 245 W}$ (wm refers to wild type to mutant) express WT p53 initially. Cre recombinase excises the wildtype cDNA to generate the mutant $\operatorname{Trp} 53^{R 172 H}$ and $\operatorname{Trp} 53^{R 245 W}$ alleles (Fig. 1a). The $\operatorname{Tr} p 53^{R 172 H}$ allele is identical to a previous germline mutant Trp53 allele ${ }^{12}$, and germline $\operatorname{Trp} 53^{R 245 W}$ mice are tumor-prone (unpublished data). Sequencing of the alleles ensured absence of other alterations (For details, please refer to the Methods section).

The Trp53 $3^{w m-R 172 H}$ and $\operatorname{Tr} p 53^{w m-R 245 W}$ alleles express a fulllength p53 protein in heterozygous mice, which is stable in response to $\gamma$-radiation (Fig. $1 \mathrm{~b}$ and Supplementary Fig. 2), and can transcriptionally activate canonical p53 targets, Cdkn1a ( $p 21)$ and Bbc3 (Puma), similar to WT p53 (Fig. 1c). In addition, thymuses of $\operatorname{Trp} 53^{w m-R 172 H /+}$ and $\operatorname{Trp5} 53^{w m-R 245 W /+}$ mice showed similar levels of apoptosis in response to $\gamma$-radiation (Fig. 1d) as WT mice. No differences in cell cycle arrest were observed between irradiated cultures of WT, Trp53wm-R172H/+ and $\operatorname{Trp} 53^{w m-R 245 W /+}$ mouse embryo fibroblasts (MEFs, Fig. 1e). $W T, \operatorname{Trp} 53^{w m-R 172 H /+}$ and $\operatorname{Trp} 53^{w m-R 245 W /+}$ mice also showed no significant differences in survival (Fig. 1f). Trp53wm-R172H/and $\operatorname{Tr} p 53^{+/-}$mice were tumor-prone as expected and exhibited similar survival as well (Fig. 1g). Thus, detailed characterization of the $\operatorname{Trp} 53^{w m-R 172 H}$ and $\operatorname{Trp} 53^{w m-R 245 W}$ alleles showed they express WT p53 that maintains normal function.

Somatic $\operatorname{Trp53}$ mutations differentially drive mammary tumors. To generate a somatic breast cancer model, we injected adenoviruses expressing Cre recombinase (Ad-Cre) into the mammary ducts of Trp53 $3^{w m-R 172 H /+}$ and Trp $53^{w m-R 245 W /+}$ mice. Modulating the dose of Ad-Cre allowed us to induce recombination of the Rosa26-LSL-tdTomato (a conditional allele that expresses tomato red fluorescent protein in response to $\mathrm{Cre}^{13}$ ), and likely $\operatorname{Trp53}$ in $1-5 \%$ (low dose) or $50-70 \%$ (high dose) of epithelial cells (Fig. 2a). None of the 21 injected Trp53wm-R172H/+ mice developed mammary tumors by 25 months post Ad-Cre injection (Fig. 2b, c and Table 1). To enhance tumor development, Ad-Cre-injected Trp53 $3^{w m-R 172 H /+}$ female mice were exposed to $\gamma$-radiation. One of the eight mice (low dose Ad-Cre) developed a mammary tumor at 23.7 months, and four of nine mice (high dose Ad-Cre) at 18.3 months on average (Fig. 2b, c and Table 1). Only one tumor metastasized to lung (Fig. 2d). All $\operatorname{Trp} 53^{w m-R 172 H /+}$ females treated with Ad-Cre and $\gamma$-radiation showed loss of the WT Trp53 allele (Fig. 2e). To examine the potential cooperative role of loss of heterozygosity (LOH), cohorts of $\operatorname{Trp} 53^{w m-R 172 H / f l}$ female mice were established, in which the conditional $\operatorname{Tr} p 53^{f l}$ allele is simultaneously deleted when $\operatorname{Trp} 53^{w m-R 172 H}$ becomes mutant in response to Cre. Remarkably, following high dose Ad-Cre injection, the Trp53 5 wm$R 172 \mathrm{H} / \mathrm{fl}$ mice developed mammary tumors with a high frequency (90\%), at around 14.7 months on average (Fig. 2b, c and Table 1). Two of 11 tumors disseminated to lung (Fig. 2d). These data suggest a strong inhibitory role of WT p53 against p53R172Hinduced mammary tumorigenesis. 
In contrast, Trp53R245W heterozygosity was sufficient for mammary tumorigenesis. Thirteen of $25 \operatorname{Trp} 53^{w m-R 245 W /+}$ mice injected with Ad-Cre at either dose developed tumors (Fig. 2b, c and Table 1) with $46 \%$ metastasis to lung and liver (Fig. 2d). p53R245W tumors showed more variation in LOH with $55.6 \%$ tumors having $\mathrm{LOH}, 22.2 \%$ tumors without $\mathrm{LOH}$, and $22.2 \%$ retaining WT alleles in some cells (Fig. 2e and Supplementary
Fig. 3). The efficiency of Cre-mediated recombination was similar for both alleles (Supplementary Fig. 4).

To increase the power to identify differences between missense mutations at TP53R175 and TP53R248 in human patients, we analyzed a combined dataset of patients who developed one of three epithelial cancers (breast, ovarian, and lung carcinomas), and found that individuals with TP53R248 mutations (tryptophan

a

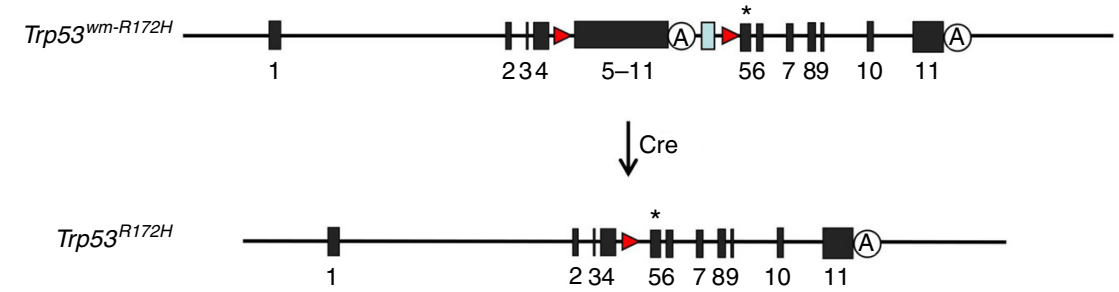

$\operatorname{Trp53}{ }^{\mathrm{wm}-\mathrm{R} 245 \mathrm{~W}}$
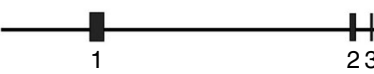

${ }_{234}$

$\downarrow$ Cre

$\operatorname{Trp53^{R245W}}$

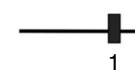

HH-HNH H A

b

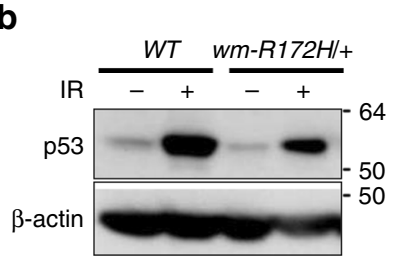

C
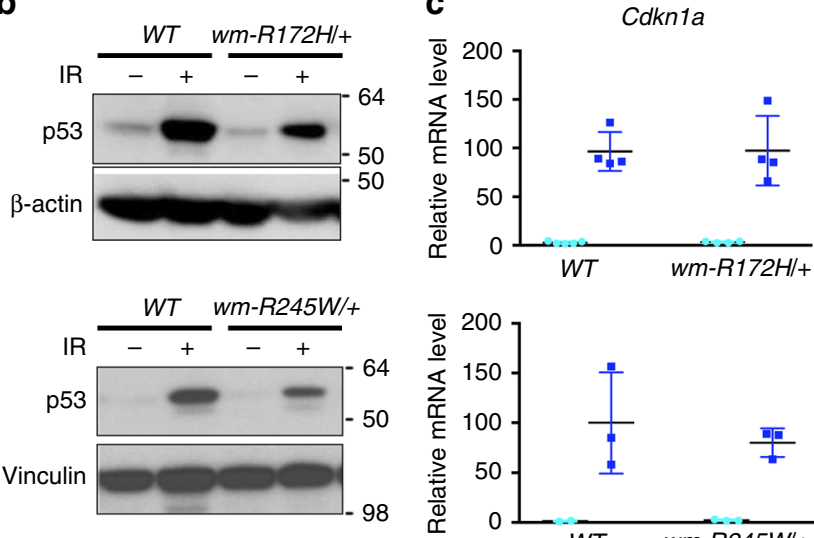

$W T$
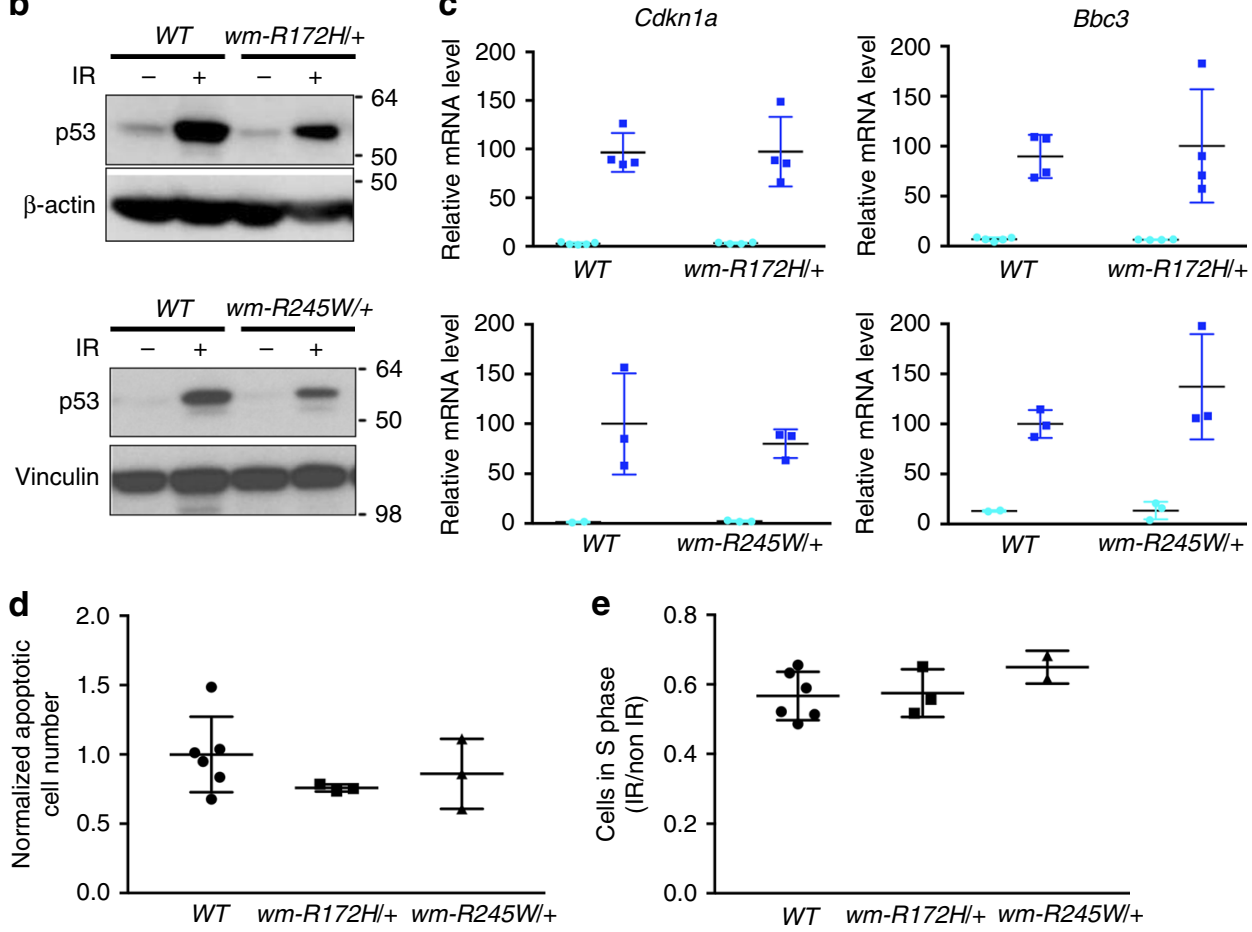

f

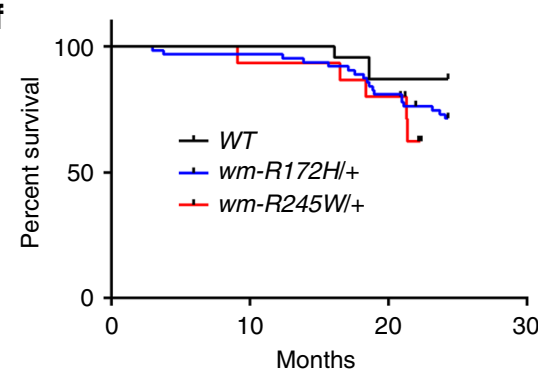

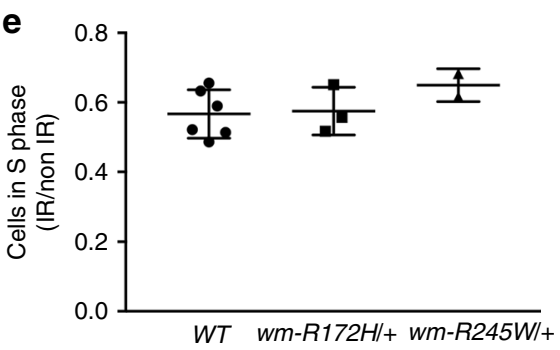

g

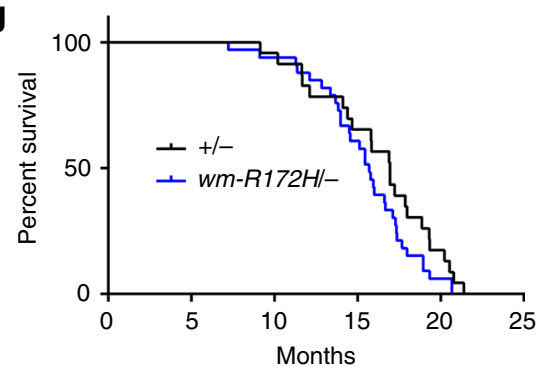




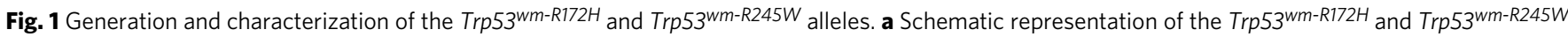
alleles. The cre-loxP-mediated strategy was used to generate the $\operatorname{Trp} 53^{R 172 H}$ and $\operatorname{Trp} 53^{R 245 W}$ alleles. Red triangle loxP site; blue rectangle Frt site left after removing the selection markers (see Supplementary Fig. 1); A, Trp53 native polyadenylation signaling sequence; ${ }^{\star} 515 \mathrm{G}->\mathrm{C}$ mutation was introduced in exon 5 for the $\operatorname{Trp} 53^{\mathrm{Wm}}-\mathrm{R} 172 \mathrm{H}$ allele, and $733 \mathrm{C} \rightarrow \mathrm{T}$ and $735 \mathrm{C} \rightarrow \mathrm{G}$ mutations were introduced in exon 7 for the Trp53wm-R245W allele. b Western blot analysis for p53 protein levels in thymuses of various Trp53 genotypes ( WT, wm- $R 172 \mathrm{H} /+$ and $w m-R 245 W /+)$. IR, $\gamma$-radiation. c Real-time reverse transcription PCR analysis for Cdkn1a and Bbc3 mRNAs in thymuses of various Trp53 genotypes (WT, wm-R172H/+ and wm-R245W/+). Light blue circles, mRNA level of Cdkn1a or Bbc3 in non-irradiated thymuses; Dark blue squares, mRNA level of Cdkn1a or Bbc3 in irradiated thymuses. Error bars, s.d. d Quantification of the normalized number of $\gamma$-radiation-induced apoptotic thymocytes of various Trp53 genotypes $(W T$, wm- $R 172 H /+$ and $w m-R 245 W /+$ ), following annexin $V$ staining and FACS analysis. Error bars, s.d. e Quantification of cells in S phase for $\gamma$-irradiated and non-irradiated MEFs of various Trp53 genotypes (WT, $w m-R 172 \mathrm{H} /+$ and $w m-R 245 W /+)$. Error bars, s.d. f Kaplan-Meier survival curves of Trp53 WT $(n=23), \operatorname{Trp53} w m-R 172 \mathrm{H} /+(n=42)$ and $\operatorname{Trp} 53^{w m}-R 245 \mathrm{~W} /+$ $(n=15)$ mice. $\mathbf{g}$ Kaplan-Meier survival curves of $\left.\operatorname{Trp53^{+}/-} n=23\right)$ and $\operatorname{Trp53}{ }^{\mathrm{Wm}-\mathrm{R} 172 \mathrm{H} /-}(n=33)$ mice. In $\mathbf{c}-\mathbf{g}$, no statistical differences were observed between samples with different Trp53 genotypes ( $W T$, wm- $R 172 \mathrm{H} /+$ and $w m-R 245 W /+$ )

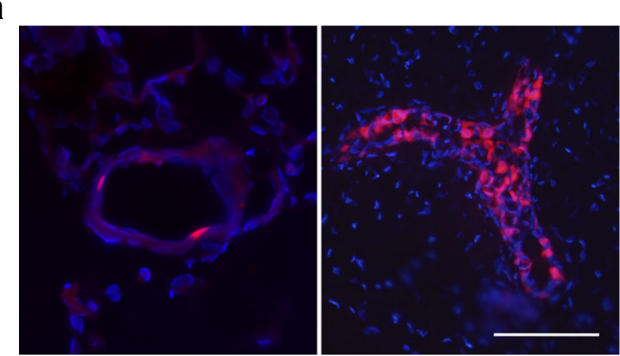

C

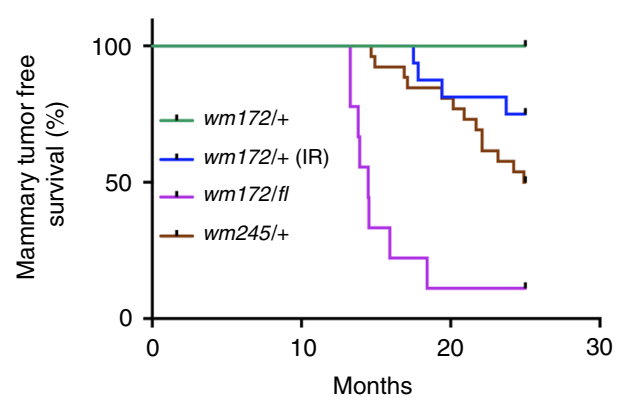

e

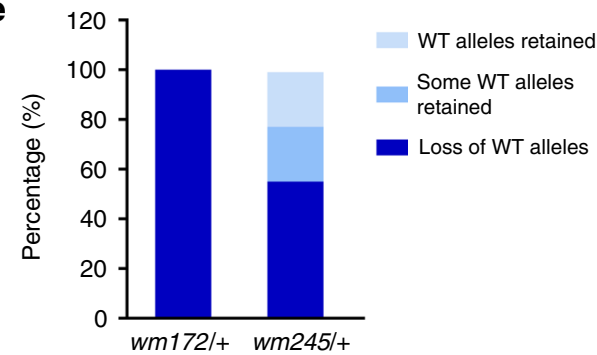

(IR)

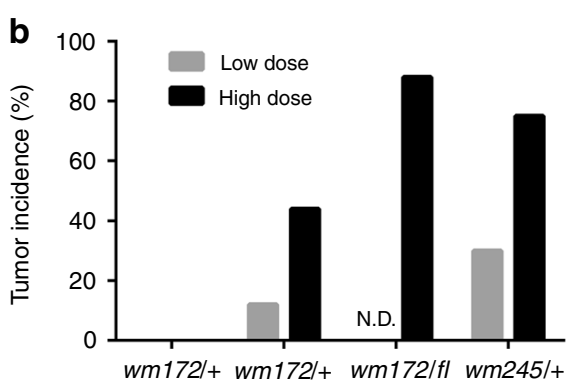

(IR)

d

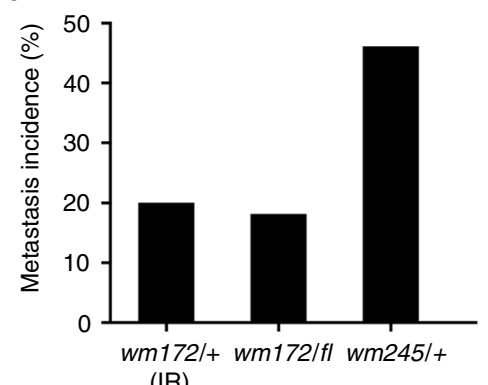

$(\mathrm{IR})$

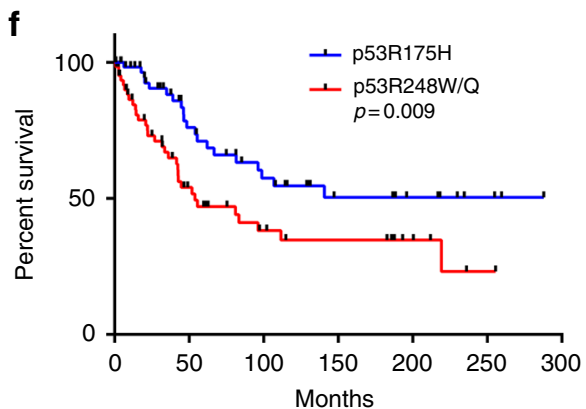

Fig. 2 Somatic Trp53R172H and Trp53R245W mutations in mouse mammary epithelium drive tumorigenesis. a Representative pictures of mouse mammary glands from Rosa26-LSL-tdTomato mice after Ad-Cre injection in mammary ducts. Two doses of Ad-Cre were deployed for mammary intraductal injection, with low dose infecting 1-5\% epithelial cells (left), and high dose 50-70\% (right). Scale bar shows $100 \mu \mathrm{m}$ and applies to both sections. b Comparison of the tumor incidences from mice of various Trp53 genotypes (wm172/+, Trp53wm-R172H/+; wm172/fl, Trp53wm-R172H/fl; wm245/+, Trp53wm-R245W/ +) after low vs. high doses of Ad-Cre injection. IR, $\gamma$-radiation. For animal numbers in each group, please refer to Table 1. N.D., non-determined. c Kaplan-Meier mammary tumor free survival curves of Ad-Cre injected mice with various Trp53 genotypes $(w m 172 /+, w m-R 172 H /+, n=21 ; w m 172 /+$ (IR), wm-R172H/+ ( $\gamma$-radiation), $n=17 ; w m 172 / f I, w m-R 172 H / f I, n=10 ; w m 245 /+, w m-R 245 W /+, n=25)$. d Comparison of metastasis incidences of mammary tumors from Ad-Cre-injected mice with various Trp53 genotypes (wm172/+ (IR), wm-R172H/+ ( $\gamma$-radiation), $n=5 ; w m 172 / f 1, w m-R 172 H / f l, n=$ 11; wm245/+, wm-R245W/,$+ n=13)$. e The status of the WT Trp53 allele in mammary tumors from Ad-Cre-injected mice with various Trp53 genotypes $(w m 172 /+(I R), w m-R 172 H /+(\gamma$-radiation $), n=5 ; w m 245 /+, w m-R 245 W /+, n=9) . \mathbf{f}$ Kaplan-Meier survival curves of human breast, ovarian and lung cancer patients containing TP53R175H $(n=62)$ or TP53R248W/Q (TP53R248W or TP53R248Q, $n=65)$ mutations. $p=0.009$ ( $t$-test) 


\begin{tabular}{|c|c|c|c|c|}
\hline $\begin{array}{l}\text { Mouse } \\
\text { genotype }\end{array}$ & Treatment & $\begin{array}{l}\text { Virus dose } \\
\text { (mouse } \\
\text { numbers) }\end{array}$ & $\begin{array}{l}\text { Tumor } \\
\text { (metastasis) }\end{array}$ & $\begin{array}{l}\text { Latency } \\
\text { (months) }\end{array}$ \\
\hline \multirow{5}{*}{$\begin{array}{l}p 53 w m- \\
R 172 \mathrm{H} /+\end{array}$} & Ad & High (2) & $0(0)$ & $\mathrm{N} / \mathrm{A}$ \\
\hline & Ad-Cre & Low (10) & $0(0)$ & $\mathrm{N} / \mathrm{A}$ \\
\hline & & High (11) & $1^{b}(0)$ & $25.4^{b}$ \\
\hline & Ad-Cre+IR & Low (8) & $1(0)$ & 23.7 \\
\hline & & High (9) & $4(1)$ & 18.3 \\
\hline $\begin{array}{l}p 53^{w m}- \\
R 172 \mathrm{H} / \mathrm{fl}\end{array}$ & Ad-Cre & High (10) & $11(2)$ & 14.7 \\
\hline \multirow{3}{*}{$\begin{array}{l}p 53^{w m-} \\
R 245 W /+\end{array}$} & Ad & High (2) & $0(0)$ & $\mathrm{N} / \mathrm{A}$ \\
\hline & Ad-Cre & Low (13) & $4(1)$ & 23.2 \\
\hline & & High (12) & $9(5)$ & 18.8 \\
\hline
\end{tabular}

aReferring to time after Ad or Ad-Cre injection

bMice were generally euthanized around 25 months after Ad-Cre injection if no tumors were formed, except for this female, which was kept for longer time accidently and exhibited a tumor at 25.4 months post Ad-Cre injection.

$\mathrm{IR}, \gamma$-radiation

or glutamine) exhibited a significant worse survival compared to ones with the TP53R175H mutation (Fig. 2f).

Pathological examination identified all tumors from $\operatorname{Tr} p 53^{w m-}$ $R 172 \mathrm{H} /+$ (irradiated) and $\operatorname{Tr} p 53^{\mathrm{wm}-\mathrm{R} 172 \mathrm{H} / \mathrm{fl}}$ mice as adenocarcinomas (Supplementary Fig. 5). In contrast, tumors from the Trp53 $3^{w m-R 245 W /+}$ mice were more diverse: adenocarcinoma (76.9\%), anaplastic carcinoma (15.4\%) and sarcomatoid carcinoma (7.7\%) (Supplementary Fig. 5), with distinct morphologies (Fig. 3a-c). Metastases exhibited morphological similarities with the associated primary tumors (Compare Fig. 3d to Fig. 3a and Fig. 3e to Fig. 3b). To identify the molecular subtypes of tumors, we measured expression of Esr1, Pgr, and Erbb2. All five mammary tumors from Trp53 $3^{\mathrm{wm}-\mathrm{R} 172 \mathrm{H} /+}$ mice (Ad-Cre plus IR) exhibited minimal level of the Esr1 and Pgr transcripts, and only one of these tumors showed higher expression of Erbb2, making them predominantly triple negative ( $80 \%$, Fig. $3 \mathrm{f}, \mathrm{g})$. In contrast, the p53R245W-driven tumors exhibited more diverse molecular subtypes: $\mathrm{Esr}^{-} \mathrm{Pgr}^{-} \mathrm{Erbb2}^{-}$(triple negative, 30.8\%), $\mathrm{Esr}^{-} \mathrm{Pgr}^{-} \mathrm{Erbb2}^{+}$(Her2 enriched, 38.4\%), or Esr1/Pgr ${ }^{+} \mathrm{Erbb2}^{+}$ (Esr1 or Pgr, or both, positive, most likely luminal B, 30.8\%) (Fig. 3f, g). The above association between specific Trp53 hot spot mutations and breast tumor subtype phenocopies human breast cancers, where p53R175 mutations were most frequently identified in triple negative breast cancer and p53R248 mutations in triple negative, Her2 enriched and luminal B breast cancers ${ }^{2}$. In the $\operatorname{Tr} p 53^{w m-R 172 H / f l}$ mice, loss of the WT allele altered the subtype spectrum of $\mathrm{p} 53 \mathrm{R} 172 \mathrm{H}$ driven tumors. The majority of tumors from these mice was Esr1/Pgr ${ }^{+} \mathrm{Erbb2}^{+}(66.7 \%)$, and the rest were Erbb2 positive (25.0\%) or $\mathrm{Esr1}^{-} \mathrm{Pgr}^{+} \mathrm{Erbb2}^{-}$(8.3\%) (Fig. 3f, g).

Previously, a panel of genes that mark or mediate human breast cancer metastasis to the lungs have been identified ${ }^{14}$. We therefore examined whether expression of 18 of these genes, which showed significance either from transcriptomic analysis or functional validation, was also enriched in the p53R245W mammary tumors, which had high metastatic incidence (Fig. 2d). Strikingly, seven genes were significantly highly expressed in p53R245W tumors as compared to the less metastatic p53R172H tumors (Fig. $3 \mathrm{~h}$ and Supplementary Fig. 6). In addition, Sparc, a matricellular glycoprotein associated with human breast cancer aggressiveness $^{14,15}$, was expressed significantly higher in metastatic versus non-metastatic p53R245W tumors (Fig. 3i). Thus, the data suggest that expression of Trp53 missense mutations in a few mammary epithelial cells results in aggressive breast cancers that share many features of human breast cancers.

Genomic heterogeneity in somatic p53R245W mammary tumors. In recent years, genomic sequencing has allowed analysis of the genetic basis of tumor progression and metastasis in different types of cancers, including breast cancer ${ }^{16}$, which may enable tailoring of therapeutic strategies to the fundamental molecular lesions driving a particular tumor. The faithful recapitulation of many features of human breast cancer makes our p53R245W mammary tumor model a useful tool for understanding invasive breast tumors driven by p53R245W. We performed whole exome sequencing of three spatially separated regions from two primary mammary adenocarcinomas and associated lung metastasis (3 for each primary tumor) from $\operatorname{Tr} 553^{w m-R 245 W /+}$ mice. All tumors and normal lung controls were sequenced at a depth averaging 96.4×. Mouse \#4 had 185 mutations present in primary tumor site(s), and 193 mutations in metastatic site(s). Mouse \#27 had 204 mutations in primary site (s) and 215 in metastatic site(s). The majority of these were missense and splice site mutations (Fig. 4a and Supplementary Data 1). Interestingly, genes that are involved in chromosome replication, segregation, or repair, such as $S m c 5^{17}$ and $R f c 1^{18}$, or well-known tumor suppressors, such as $\operatorname{Pten}^{19}$, were among the list. It is also noteworthy that in addition to the $\operatorname{TrP} 53$ mutation, there were 15 other mutations that were shared among all sequenced samples (Fig. 4a and Supplementary Data 1) for each mouse. These mutations may play critical cooperative roles in p53R245W-initiated tumorigenesis and further validation is needed.

Analysis of intra-tumor heterogeneity of samples revealed 19 and 23 mutations shared among three regions sampled in primary tumors for mice \#4 and \#27, respectively, equal to $10.3 \%$ and $11.3 \%$ of total mutations identified (Supplementary Fig. 7a). Plus, $8.8 \%(17 / 193)$ and $8.4 \%(18 / 215)$ of mutations were shared among three metastatic sites, respectively (Supplementary Fig. 7b). Among 319 mutations identified for both primary tumor and metastases in mouse \#4, only 59 (18.5\%) were shared between any primary tumor site and any metastatic site (Fig. 4b). Similarly, for mouse \#27, 21.4\% (74/345) mutations were shared between any primary tumor site and any metastatic site (Fig. 4b). These shared mutations are defined as early mutations since they most likely originated in primary sites. In addition, we identified 134 and 141 mutations for \#4 and \#27, respectively, which were exclusively present in metastases and therefore were acquired after metastatic cells left the primary site and designated as late mutations. $27.1 \%$ $(16 / 59)$ and $23.0 \%(17 / 74)$ early mutations were shared among three metastatic sites in \#4 and \#27 mice, respectively (Fig. 4c). In striking contrast, there was only $0.75 \%(1 / 134)$ shared late mutations in mouse \#4 and $0.71 \%$ in mouse \#27 (1/141) (Fig. 4d). These results suggest tumor cells with metastatic potency preexist in the parental tumors.

Inter-patient heterogeneities have long been observed in different human cancer types ${ }^{16}$. Among the mutations identified in primary tumors from mouse \#4 and \#27, 13.4\% were shared between the two (Supplementary Fig. 7c, Left). In addition, 9.97\% were shared between metastatic tumors from these mice (Supplementary Fig. 7c, Right).

Mutation evolution in somatic R245W mammary tumors. Multi-region exome sequencing also allows delineating the clonal and sub-clonal evolutionary architecture of primary tumors and metastasis $^{20,21}$. All specimens from primary and metastatic 
a

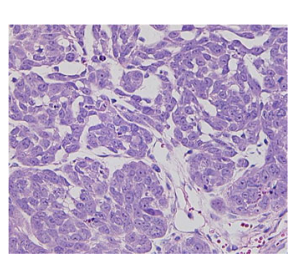

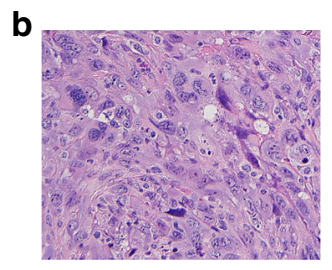
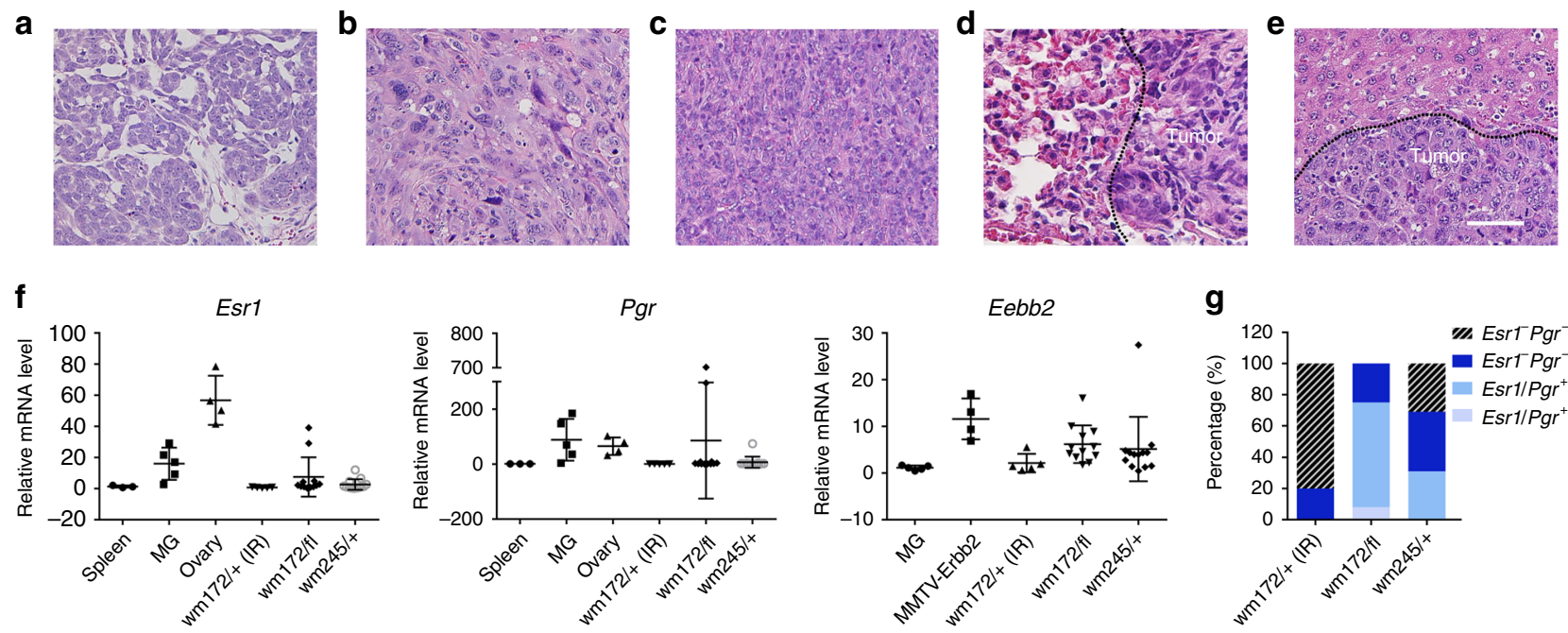

g
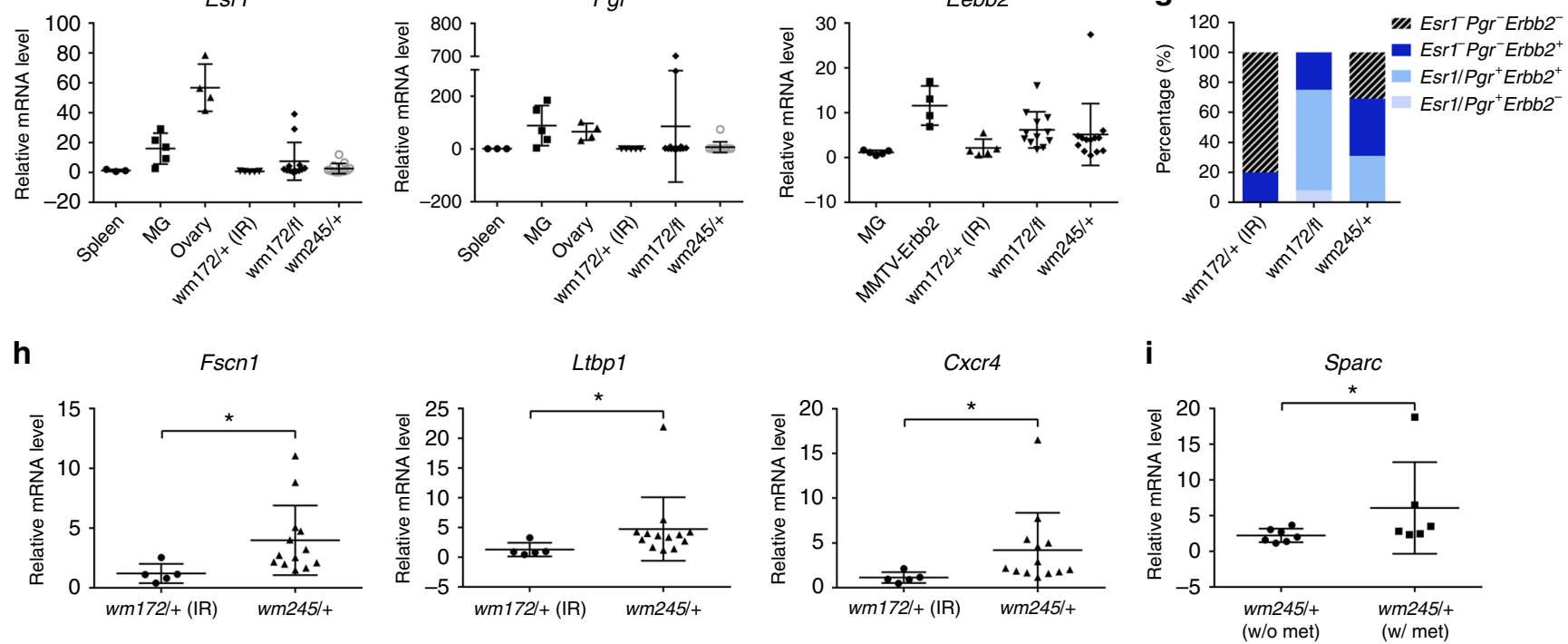

Fig. 3 Characteristics of the p53R172H and p53R245W mammary tumors a-e Hematox;ylin and Eosin-stained sections of mammary tumors from mice showing different pathological subtypes. a A moderately-differentiated adenocarcinoma. Note glandular and nesting formation of tumor composition; $\mathbf{b}$ A highly anaplastic cellular manifestation including spindle cell, cellular pleomorphism and loss of glandular and differentiation features; c A sarcomatoid carcinoma with an undifferentiated sarcoma-like manifestation. d A localized metastatic adenocarcinoma in lung. Tumor nodule displays glandular proliferation of well-to-moderately-differentiated adenocarcinoma of mammary origin; e A metastatic tumor to liver. The tumor displays the same phenotypic anaplasia seen in primary tumor (b). Note the distinct boundary of metastases and normal tissues, as marked by the dashed line. Scale bar is $50 \mu \mathrm{m}$ and applies to all sections. f RT-qPCR analysis for Esr1, Pgr and Erbb2 in WT mouse spleens, mammary glands (MG), or ovaries, or mammary tumors from the MMTV-Erbb2 mice, and tumors from Ad-Cre-injected mice of various Trp53 genotypes (wm172/+, wm- $\mathrm{R} 172 \mathrm{H} /+; \mathrm{wm} 172 / \mathrm{fl}, \mathrm{wm}-\mathrm{R} 172 \mathrm{H} / \mathrm{fl} ;$ $w m 245 /+, w m-R 245 W /+$ ). IR, $\gamma$-radiation. Error bars, s.d. $\mathbf{g}$ Summary of the molecular subtypes of mammary tumors from mice of various genotypes, based on data in f. Esr1/Pgr+ label means Esr1 postive or Pgr positive, or both. h RT-qPCR analysis for Fscn1, Ltbp1, and Cxcr4, in Trp53R245W-driven mammary tumors compared to Trp53R172H mammary tumors. Error bars, s.d. * $p<0.05$ ( $t$-test) i RT-qPCR analysis for Sparc in Trp53R245W-driven mammary tumors that had metastasis compared to those without metastasis. Error bars, s.d. * $p<0.05$ ( $t$-test)

lesions showed consistent truncal mutations of similar length suggesting that both tumors shared a similar timing of subclonal progression (Fig. 4e). Branching revealed two different evolving patterns of metastatic progression from primary lesions. Two metastatic sites, M1 and M2, in mouse \#4 diverged away from the primary tumor early even before the phylogenetic diversification of the primary tumor. However, the third metastatic site, M3, did not emerge until the primary tumor diversified. M3 shared the highest percentage of mutations with the primary subclone P3 (Fig. 4e, left), suggesting P3 was the origin of this metastatic site. Thus, the mammary tumor from mouse \#4 exhibits a multi-origin metastatic pattern. In mouse \#27, all three metastatic sites, M1-M3, diverged away from the primary tumor early before the primary tumor diversified phylogenetically, with M2 requiring the fewest number of mutations to depart and M3 the highest (Fig. 4e, right).

One might argue that the above multi-origin of metastases is due to independent seeding coming from individual primary tumors that interdigitate together. However, the fact that 15 truncal mutations were shared among all samples for both mice (Fig. 4a and Supplementary Data 1) argues against two entirely independent lineages generated from different initiating cells. The primary and metastases shared similar allelic frequencies for the majority of the truncal mutations (Supplementary Fig. 8), further supporting the scenario where only one primary clone exists.

The above findings are clinically relevant and indicate that treating actionable mutations that are subclonal in the primary tumor may not prevent disease relapse. The extremely early dissemination observed in mammary tumors from mouse \#4 and \#27 also warrants the necessity of closely monitoring breast cancer patients containing the TP53R248W mutation even when the primary lesion is discovered and treated at a very early stage.

\section{Discussion}

The strikingly high frequency of TP53 mutation in human cancers and early studies showing the transformation ability of mutant TP53 in cultured cell lines make it unquestionably critical to examine outcomes in genetically modified mice. In our current study, we generated two conditional $\operatorname{Trp} 53^{w m-R 172 H}$ and Trp53 $3^{w m-R 245 W}$ alleles, which allow mutant p53 expression upon Cre-mediated recombination. Importantly, in the absence of Cre, the Trp53wm-R172H and Trp53 $3^{w m-R 245 W}$ alleles express functional WT p53, leading to several advantages over all other conditional mutant $\operatorname{Trp} 53$ alleles. 1) the $\operatorname{Trp} 53^{\mathrm{wm} /+}$ mice express normal levels of WT p53 and are not tumor prone; 2) mutant p53 is 
a

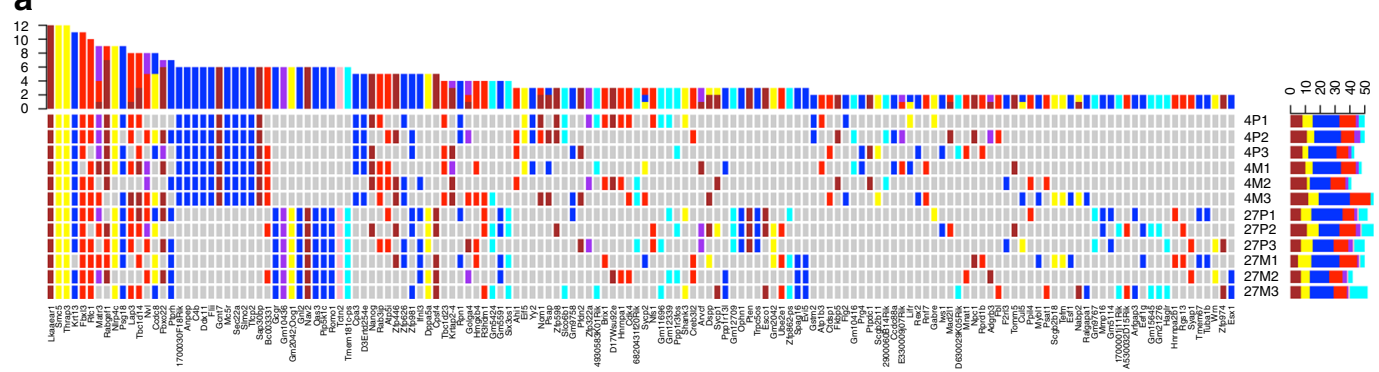

Missense Deletion

Insertion _ Stop

- Splicing

ncRNA-exonic Unknown

b

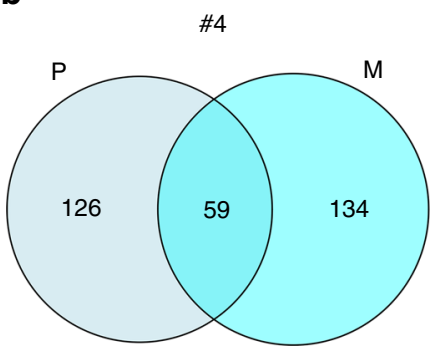

\#27

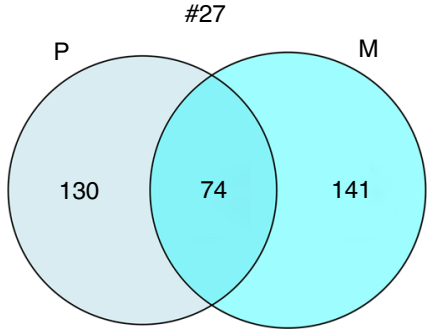

C
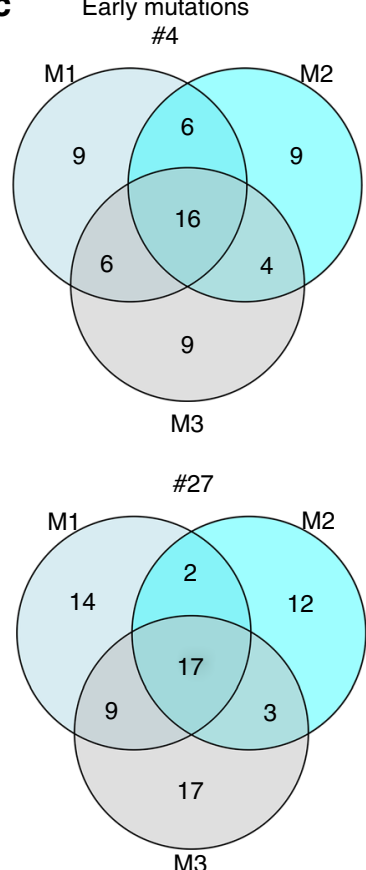

d Late mutations

\#4

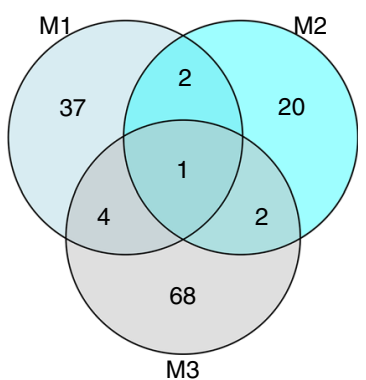

\#27

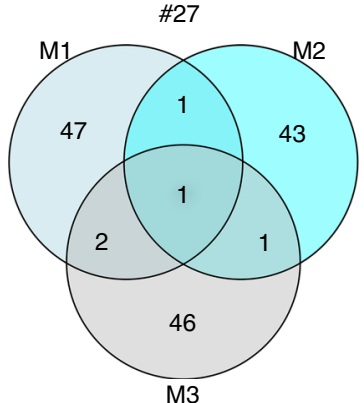

e

\#4

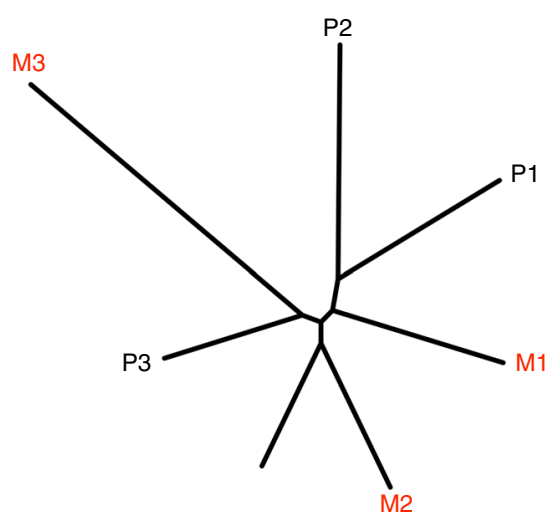

\#27

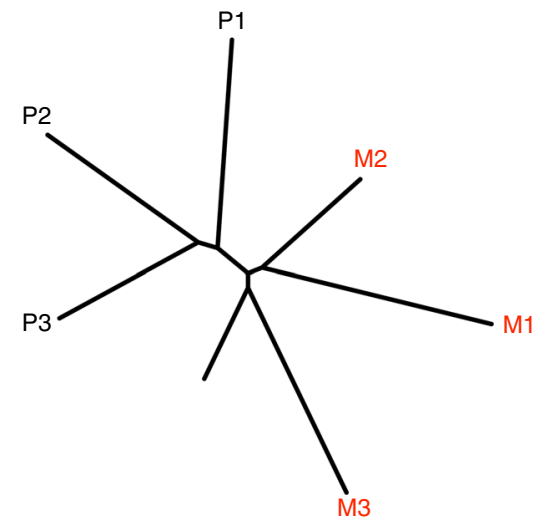

Fig. 4 Genomic heterogeneity and mutational evolution in mammary tumors driven by somatic Trp53R245W mutant. a Heatmaps of genes with various alterations identified in at least two tumors or metastases of the three physically separated regions of primary tumors (P1-P3) and associated metastatic clones (M1-M3) from each of two Trp53wm-R245W/+ mice (\#4 and \#27), subjected to high dose Ad-Cre intraductal injection. Note that some genes have more than one alterations. $\mathbf{b}$ Venn diagrams depicting the overlap of the identified mutations in primary tumors ( $P$ ) with those in the associated metastases $(M)$ in mouse \#4 and \#27. c Venn diagrams depicting the overlap of the identified early mutations among all three metastatic clones (M1-M3) in mouse \#4 and \#27, respectively. $\mathbf{d}$ Venn diagrams depicting the overlap of the identified late mutations among all three metastatic clones (M1-M3) in mouse \#4 and \#27, respectively. e Phylogenetic trees generated by clustering genome-wide mutation data from 3 multi-region primary tumor samples (P1-P3) and 3 metastatic clones (M1-M3) for both mouse \#4 and \#27. Relative branch lengths were determined from the proportion of mutations in each branch 
converted from WT at the endogenous locus closely mimicking the clinical situation; 3) mutant p53 is induced in the specific tissue/cell of interest, limiting tumor development to a single tissue; 4) the tumor microenvironment and immune system of the animals are p53 WT.

The mammary tumors that developed from our models recapitulated a number of characteristics of human breast cancers. Tumors from the $\operatorname{Tr} p 53^{w m-R 172 H /+}$ mice were largely triple negative, whereas those from the $\operatorname{Tr} p 53^{w m-R 245 W /+}$ mice were triple negative, Her2 positive, and luminal B, faithfully phenocoping the association of different TP53 hot spot mutations with specific breast cancer subtypes in humans ${ }^{2}$. In addition, the $\operatorname{Trp} 53 R 245 \mathrm{~W}$ mutation led to highly metastatic mammary tumors correlating to shortened survival in patients with epithelial cancers that contain TP53R248 mutations. Moreover, some, although not all, genes identified previously to mark human breast cancer metastasis to lung were also highly expressed in the p53R245W driven mammary tumors that had higher lung metastasis incidence compared to p53R172H tumors.

Emerging in vivo evidence shows that hot spot $p 53$ mutations are not functionally equal. For example, germline $\operatorname{Tr} p 53 \mathrm{R} 172 \mathrm{H}$ and $\operatorname{Trp} 53 \mathrm{R} 270 \mathrm{H}$ mice exhibited significant differences in their tumor spectra ${ }^{22}$. In addition, in human TP53 Knock-in (HUPKI) models expressing the p53R248Q and p53G245S mutants, $T P 53^{R 248 \mathrm{Q} /}$ mice showed decreased survival, faster tumor development, and a broader tumor spectrum when compared to TP53 ${ }^{\mathrm{G} 245 \mathrm{~S} /-}$ animals $^{23}$. In our study, somatic p53R172H was less potent in initiating mammary tumorigenesis compared to p53R245W, and the p53R245W tumors had a higher frequency of metastasis. It will also be interesting to investigate whether and how these p53 mutants differentially impact tumor cells in drug responses/resistance. It is not clear why these hot spot $\operatorname{Trp} 53$ mutations exhibit different magnitudes of effects. However, it is noteworthy that the above-mentioned differences indicate that p53 structural mutants (mouse p53R172H and human p53G245S) are less potent than contact mutants (mouse p53R245W, and human p53R248Q). We speculate that, due to their different protein structures, these two categories of p53 mutants may have a distinct binding partners, which may differentially modify their impact. Previously, it has been shown that p53 mutants regulate gene expression through interacting with transcriptional factors ${ }^{24}$. A comparison of the transcriptional targets of these different p53 mutants in our breast tumors with chromatin immunoprecipitation combined with sequencing, and/ or 3'-RNA sequencing is needed. Furthermore, in contrast to p53 contact mutants, the p53 structural mutants, due to their drastic structure alterations, may have a weaker interaction with the WT p53 and therefore lead to a dampened dominant-negative effect. This speculation is supported by our observation that all the p53R172H mammary tumors examined underwent $\mathrm{LOH}$, while the WT $\operatorname{Trp} 53$ allele was retained in some p53R245W tumors.

The evident metastasis frequency of the mammary tumors driven by $\operatorname{Trp} 53 R 245 \mathrm{~W}$ mutation also allowed us to expand our understanding of the metastatic paradigm. So far, two patterns have been proposed for metastasis evolution ${ }^{25}$. Parallel evolution, where seeding from an ancestral clone occurs early during disease progression, resulting in a branched pattern of evolution. In contrast, when spreading occurs late in the evolution of the primary tumor, a linear pattern of evolution is observed. In our study, two p53R245W tumors displayed parallel evolution with a main trunk and distinct splits in both cases. Remarkably, in mouse \#27, all the metastatic clones diverge away from the primary tumor before the subclonal diversification of the primary lesion occurs, indicating extremely early dissemination. This finding has important clinical implications. In contrast to tumors with linear evolution, patients with tumors that evolve in a branched fashion, e.g., TP53R248W breast tumors, may require closer monitoring even when the primary lesion is discovered and treated at a very early stage, due to the early spreading of tumor cells.

It is not clear why different evolution patterns exist among different tumors. One can speculate that it may reflect the intrinsic property of the initial oncogenic driver(s). Strong oncogenic driver(s), e.g., Trp53R245W in our study, after inducing a handful of mutations and/or genomic instability, may have endowed tumor cells sufficient metastatic capability so that further mutations are not required for those cells to depart the primary site. In fact, early systemic spread has also been observed in mouse breast tumors driven by Her2 or polyomavirus-middle $T$, two well-known aggressive oncogenes ${ }^{26}$. In contrast, in the linear evolution model, tumor cells in the primary disease initiated by relatively weak driver(s) probably demand a large repertoire of mutations, which takes considerable time to accumulate, before they gain the ability to spread.

These invaluable models highlight the aggressive nature of $p 53$ somatic mutations and the parallel tumor evolution pattern driven by a $p 53$ missense mutation as the initiating event. In addition to studying breast cancers, the $\operatorname{Tr} p 53^{w m-R 172 H}$ and $\operatorname{Tr} p 53^{w m-}$ $R 245 \mathrm{~W}$ alleles can also be used to investigate the role of $\mathrm{p} 53$ mutations in driving other cancers. Additionally, they can be used to determine how p53 mutant cells modify and shape their microenvironment. Mouse models will provide a better understanding of the mechanisms underlying tumor initiation and progression, which will lead to more rational drug development and testing.

\section{Methods}

Generation of Trp53 ${ }^{w m-R 172 H}$ and Trp53 ${ }^{\text {wm-R245W }}$ mouse alleles. The targeting vectors were generated by cloning a cDNA fragment that contained exons 5-11 (for the $\operatorname{Trp} 53^{w m-R 172 H}$ allele) or 7-11 (for the $\operatorname{Trp} 53^{w m-R 245 W}$ allele), as well as a polyadenylation signal, followed by an Frt-flanked PGKpuro cassette into intron 4 (for the $\operatorname{Tr} p 53^{w m-R 172 H}$ allele) or an Frt-flanked PGKneo cassette into intron 6 (for the $\operatorname{Trp} 53^{w m-R 245 W}$ allele) of a $\operatorname{Trp} 53$ genomic fragment. The cDNA fragment and PGKpuro or PGKneo cassette was flanked by loxp sequences. An arginine-tohistidine substitution (CGC to CAC) at codon 172 or arginine-to-tryptophan substitution (CGC to TGG) at codon 245 was performed by site-directed mutagenesis for the $\operatorname{Tr} p 53^{w m-R 172 H}$ and $\operatorname{Tr} p 53^{w m-R 245 W}$ alleles, respectively (details in Supplementary Fig. 1a). The resulting constructs were then individually cloned into a vector containing a thymidine kinase (TK) cassette. The entire targeting vectors were sequence verified, linearized with NotI, and electroporated into AB-1 embryonic stem (ES) cells. DNA from G418-resistant FIAU-sensitive ES colonies was subjected to Southern blot analysis (Supplementary Fig. 1c, d and Supplementary Fig. 9). For each allele, two targeted ES cell clones were injected into $\mathrm{C} 57 \mathrm{BL} / 6$ blastocysts and transferred into pseudo pregnant CD1 female recipients. The resulting chimeras were mated with $\mathrm{C} 57 \mathrm{BL} / 6$ females to secure germline transmission of the mutant alleles. Southern blot analysis was performed to reassure correct targeting in F1 mice, or a combination of primers internal and external to the targeting vectors was used to amplify the alleles and PCR products were sequenced to ensure absence of other alternations. Mice with the targeted alleles were crossed with Rosa26-flp mice (also in the C57BL/6 background) to remove the PGKpuro or PGKneo cassettes. The resulting mice were maintained in a mixed C57BL/6/129 S strain background. All mouse experiments were carried out in compliance with the guidelines of the American Association for Accreditation for Laboratory Animal Care International and the US Public Health Service Policy on Humane Care and Use of Laboratory Animals. Study protocols were approved by M.D. Anderson Cancer Center Institutional Animal Care and Use Committee.

Genotyping. Genotyping was performed by polymerase chain reaction (PCR) using primers surrounding the $5^{\prime}$ loxp site for both alleles. The primers used to genotype both alleles were as follows: $\operatorname{Tr} p 53^{w m-R 172 H}$ forward, $5^{\prime}$-AGCTGCTAGAGACAGTTGAGG-3', reverse, 5'-GCCAGCAAAGAGAGGACT-3'; Trp53 ${ }^{\mathrm{wm}-}$ $R 245 W$ forward, $5^{\prime}$-ACCTTATGAGCCACCCGA-3', reverse: 5'-GGAAGACACAGGATCCAGGT-3'.

Cell culture, apoptosis and EDU assays. MEFs were generated by crossing the appropriate mice and collecting embryos at $13.5 \mathrm{dpc}$. MEFs were maintained in Dulbeco's modified Eagle's medium (DMEM) supplemented with $10 \%$ fetal bovine serum (FBS) and penicillin $(100 \mathrm{IU} / \mathrm{ml}) /$ streptomycin $(100 \mathrm{mg} / \mathrm{ml})$. Apoptosis was measured in freshly isolated thymocytes from irradiated mice $4 \mathrm{~h}$ after $6 \mathrm{~Gy}$ 
radiation, using the annexin- $\mathrm{V}$ assay (Clontech) with fluorescence-activated cell sorting (FACS) analysis. EdU labeling and flow cytometry were performed following the manufacturer's specifications (ThermoFisher Scientific). Briefly, MEFs of various genotypes, $4 \mathrm{~h}$ after $6 \mathrm{~Gy} \gamma$-radiation, were labeled with EdU followed by fixation. Incorporated Edu was quantified by FACS analysis.

Western blot assay and reverse transcription PCR. Tissues were homogenized and protein lysates were prepared for western blots. The antibodies used were: p53, 1:1000 (NCL-L-p53-CM5p, Leica ${ }^{27}$ ), $\beta$-actin, 1:10000 (A5441, Sigma-Aldrich ${ }^{28}$ ) and Vinculin, 1:5000 (V9131, Sigma-Aldrich ${ }^{29}$ ). For full Western blot images, please refer to Supplementary Fig. 10. Total RNA was extracted from the tissues using TRIzol reagent (Life Technologies). The first-strand cDNA synthesis kit (GE Healthcare) was used for reverse transcriptase reactions. Reverse transcription quantitative PCR (RT-qPCR) was performed according to the manufacturer's specifications (Life Technologies). Data were normalized to $\beta$-actin or Rplp0. Primers used for RT-qPCR of Rplp0, Cdkn1a (p21) and Bbc3 (Puma) were as follows: $R p l p 0$ forward, 5'-CCCTGAAGTGCTCGACATCA-3', reverse, 5'-TGCGGACAC CCTCCAGAA-3'; Cdknla forward, 5'-CCTGACAGATTTCTATCACTCCA-3', reverse, 5'-CAGGCAGCGTATATCAGGAG-3'; Bbc3 forward, 5'-GCGGCGGAG ACAAGAAGA-3', reverse 5'-AGTCCCATGAAGAGATTGTACATGAC- $3^{\prime} ; 3^{\prime}, 31$. Primers used for RT-qPCR of other targets were as follows: Fscn1 forward, $5^{\prime}$-CA GCCAATCAGGATGAAGAG-3', reverse, 5'-CACCACTCGATGTCAAAGTA G-3'; Ltbp1 forward, 5'-GAGAGTCCAGGAAGGATACA-3', reverse, 5'-GCTTG TCGGATGGTATGTAG-3'; Cxcr4 forward, 5'-TAACCACCACGGCTGT A-3', reverse, 5'-CGACTATGCCAGTCAAGAAG-3'; Sparc forward, 5'-ACTCTT CCTGCCACTTCT-3', reverse, 5'-GTTGTTGCCCTCATCTCTC-3'; Man1a1, forward, 5'-ATGCACTTGTCCCACTTATC-3', reverse, 5'-GCTTCGAGATCTG TCTTGTC-3'; Nedd 9 forward, 5'-TACGAGTACCCATCCAGATAC-3', reverse, 5'-GATGGTGGAATGGCATAGAC-3'; Mmp2 forward, 5'-AGGACTATGACCG GGATAAG-3', reverse, 5'-TCCGGTCATCATCGTAGTT-3'.

Mouse mammary gland intraductal injection. Mice were crossed to $\mathrm{BALB} / \mathrm{c}$ strain to obtain Trp53 $53^{w m-R 172 H /+}$ and Trp53 5 wm-R245W/+ female mice that were $50 \%$ $\mathrm{BALB} / \mathrm{c}$ and $50 \%$ mixed C57BL/6/129 S strain backgrounds. Mice of 9-12 weeks old were anaesthetized with Ketamine and Xylazine $(10 \mu \mathrm{g}$ and $1 \mu \mathrm{g}$ per gram of body weight, respectively). Adenoviri expressing Cre (Baylor College of Medicine) were diluted in injection medium (phosphate-buffered saline supplemented with $0.1 \%$ trypan blue) and introduced into mammary ducts of the \#4 inguinal glands (10 $\mu \mathrm{l} /$ gland), using a $50 \mu \mathrm{l}$ syringe with a 30 gauge needle (Hamilton). Two doses of Ad-Cre were deployed, $5 \times 10^{4}$ particles per $\mu \mathrm{l}$ and $5 \times 10^{6}$ particles per $\mu \mathrm{l}$, to infect around $1-5 \%$ or $50-70 \%$ mammary epithelial cells, respectively. All mice were injected with the same batch adenoviri to avoid batch-to-batch variation.

LOH. LOH was examined by polymerase chain reaction (PCR) analysis using primers surrounding the mutated site in exon 5 of the Trp53wm-R172H and exon 7 of the $\operatorname{Trp} 53^{w m-R 245 W}$ alleles, respectively, followed by sequencing. The primers used to measure $\mathrm{LOH}$ in the $\operatorname{Tr} p 53^{w m-R 172 H /+}$ mice were previously described: ${ }^{30}$ forward: 5'-TACTCTCCTCCCCTCAATAAGCTATTC-3' and reverse: $5^{\prime}$-AGTCCTAACCCCACAGGCGGTGTT-3'; and for Trp53 $3^{w m-R 245 W /+}$ mice were: forward, 5'-CGGTTCCCTCCCATGCTA-3', reverse: 5'-AGCGTTGGGCATGTGGTA-3'. The criteria for determining the status of WT Trp53 alleles are described in Supplementary Fig. 3.

Histology. Tissues were fixed in $10 \% \mathrm{v} / \mathrm{v}$ formalin and embedded in paraffin. Sections were stained with haematoxylin and eosin (H\&E).

Patient survival analysis. The patient survival data were analyzed by the "Survival" R package that was downloaded from https://cran.rstudio.com/bin/macosx/ mavericks/contrib/3.3/survival_2.41-3.tgz. The survival data were retrieved from the cBioportal for Cancer Genomics website. Tumor sample IDs were extracted based on the somatic mutations R175H, R248Q, and R248W for TP53 gene. The clinical survival time and patient survival status were further retrieved from the cBioportal database based on the patient IDs. Five cancer datasets from cBioportal were used for the survival analysis: Pan-Lung Cancer (TCGA) ${ }^{32}$, Breast Cancer $(\text { METABRIC) })^{33,34}$, Breast Invasive Carcinoma (TCGA, Provisional) ${ }^{35}$, Ovarian Serous Cystadenocarcinoma (TCGA, Provisional) and Ovarian Serous Cystadenocarcinoma (TCGA) ${ }^{35}$

Whole exome sequencing and the mutation calling. DNA was extracted from mouse samples at multiple tumor regions and submitted for the exome-seq sequencing at average $96.4 \times$ coverage with Illumina Hi-seq 3000 . The raw sequencing reads were mapped to mouse genome reference (GRCm38/mm10) with BWA alignment tool ${ }^{36}$. The aligned data were further subject to the processing steps as following: sorting, duplicates marking, base recalibration, generation of panel of normal and mutation calling. The analytical tools GATK and Mutect2 developed by the Broad Institute ${ }^{37}$ were utilized. The annotation of the somatic mutations was performed with ANNOVAR tool ${ }^{38}$. The mouse snp138 database and exome sequencing of three normal mouse lungs were used to filter the mouse single nucleotide polymorphisms.

The phylogenetic analysis. The somatic mutations from primary and metastatic specimens were analyzed with the bioinformatics tool PHYLIP (Phylogeny Inference Package). The discrete character parsimony program (PARS) in PHYLIP package generated a phylogenetic tree. The tree was visualized by the drawtree function in PHYLP package.

Statistical analysis. The number of biological replicates used for comparison is indicated in each figure. Student's $t$-test was performed with Prism 6 (GraphPad Software, San Diego, CA, USA). Differences were considered significant at a value of $p<0.05$.

\section{Data availability}

Raw FASTQ sequences have been deposited to NCBI Sequence Read Archive (SRA). The accession code is SRP155491. The data that support the findings of this study are available from the corresponding authors upon reasonable request.

Received: 10 August 2017 Accepted: 15 August 2018

Published online: 27 September 2018

\section{References}

1. Dai, X. et al. Breast cancer intrinsic subtype classification, clinical use and future trends. Am. J. Cancer Res. 5, 2929-2943 (2015).

2. Silwal-Pandit, L. et al. TP53 mutation spectrum in breast cancer is subtype specific and has distinct prognostic relevance. Clin. Cancer Res. 20, 3569-3580 (2014)

3. Xiong, S. et al. Pla2g16 phospholipase mediates gain-of-function activities of mutant p53. Proc. Natl Acad. Sci. USA 111, 11145-11150 (2014).

4. Bouaoun, L. et al. TP53 variations in human cancers: new lessons from the IARC TP53 database and genomics data. Hum. Mutat. 37, 865-876 (2016).

5. Li, B. et al. A transgenic mouse model for mammary carcinogenesis. Oncogene 16, 997-1007 (1998).

6. Li, B. et al. Preferential overexpression of a 172Arg-- > Leu mutant p53 in the mammary gland of transgenic mice results in altered lobuloalveolar development. Cell Growth Differ. 5, 711-721 (1994).

7. Wijnhoven, S. W. et al. Mice expressing a mammary gland-specific R $270 \mathrm{H}$ mutation in the p53 tumor suppressor gene mimic human breast cancer development. Cancer Res. 65, 8166-8173 (2005).

8. Jackson, E. L. et al. The differential effects of mutant p53 alleles on advanced murine lung cancer. Cancer Res. 65, 10280-10288 (2005).

9. Kirsch, D. G. et al. A spatially and temporally restricted mouse model of soft tissue sarcoma. Nat. Med. 13, 992-997 (2007).

10. Cui, Y. \& Guo, G. Immunomodulatory function of the tumor suppressor p53 in host immune response and the tumor microenvironment. Int. J. Mol. Sci. 17, 1942 (2016).

11. Hill, R., Song, Y., Cardiff, R. D. \& Van Dyke, T. Selective evolution of stromal mesenchyme with p53 loss in response to epithelial tumorigenesis. Cell 123, 1001-1011 (2005)

12. Lang, G. A. et al. Gain of function of a p53 hot spot mutation in a mouse model of Li-Fraumeni syndrome. Cell 119, 861-872 (2004).

13. Madisen, L. et al. A robust and high-throughput Cre reporting and characterization system for the whole mouse brain. Nat. Neurosci. 13, 133-140 (2010).

14. Minn, A. J. et al. Genes that mediate breast cancer metastasis to lung. Nature 436, 518-524 (2005).

15. Zhu, A. et al. SPARC overexpression in primary tumors correlates with disease recurrence and overall survival in patients with triple negative breast cancer. Oncotarget 7, 76628-76634 (2016)

16. Vogelstein, B. et al. Cancer genome landscapes. Science 339, 1546-1558 (2013).

17. Kegel, A. \& Sjogren, C. The Smc5/6 complex: more than repair? Cold Spring Harb. Symp. Quant. Biol. 75, 179-187 (2010).

18. Fotedar, R. et al. A conserved domain of the large subunit of replication factor $\mathrm{C}$ binds PCNA and acts like a dominant negative inhibitor of DNA replication in mammalian cells. EMBO J. 15, 4423-4433 (1996).

19. Steck, P. A. et al. Identification of a candidate tumour suppressor gene, MMAC1, at chromosome 10q23.3 that is mutated in multiple advanced cancers. Nat. Genet. 15, 356-362 (1997)

20. Gerlinger, M. et al. Intratumor heterogeneity and branched evolution revealed by multiregion sequencing. N. Engl. J. Med. 366, 883-892 (2012).

21. Yates, L. R. et al. Subclonal diversification of primary breast cancer revealed by multiregion sequencing. Nat. Med. 21, 751-759 (2015). 
22. Olive, K. P. et al. Mutant $\mathrm{p} 53$ gain of function in two mouse models of LiFraumeni syndrome. Cell 119, 847-860 (2004).

23. Hanel, W. et al. Two hot spot mutant p53 mouse models display differential gain of function in tumorigenesis. Cell Death Differ. 20, 898-909 (2013).

24. Kim, M. P. \& Lozano, G. Mutant p53 partners in crime. Cell Death Differ. 25, 161-168 (2018).

25. Macintyre, G. et al. How subclonal modeling is changing the metastatic paradigm. Clin. Cancer Res. 23, 630-635 (2017).

26. Husemann, Y. et al. Systemic spread is an early step in breast cancer. Cancer Cell 13, 58-68 (2008).

27. Botchkarev, V. A. et al. p53 Involvement in the control of murine hair follicle regression. Am. J. Pathol. 158, 1913-1919 (2001).

28. Takahashi, K. \& Yamanaka, S. Induction of pluripotent stem cells from mouse embryonic and adult fibroblast cultures by defined factors. Cell 126, 663-676 (2006).

29. Rubashkin, M. G. et al. Force engages vinculin and promotes tumor progression by enhancing PI3K activation of phosphatidylinositol $(3,4,5)$ triphosphate. Cancer Res. 74, 4597-4611 (2014).

30. Post, S. M. et al. A high-frequency regulatory polymorphism in the p53 pathway accelerates tumor development. Cancer Cell 18, 220-230 (2010).

31. Iwakuma, T. et al. Mutation at p53 serine 389 does not rescue the embryonic lethality in $\mathrm{mdm} 2$ or $\mathrm{mdm} 4$ null mice. Oncogene 23, 7644-7650 (2004).

32. Campbell, J. D. et al. Distinct patterns of somatic genome alterations in lung adenocarcinomas and squamous cell carcinomas. Nat. Genet. 48, 607-616 (2016).

33. Cancer Genome Atlas, N.. Comprehensive molecular portraits of human breast tumours. Nature 490, 61-70 (2012).

34. Pereira, B. et al. The somatic mutation profiles of 2433 breast cancers refines their genomic and transcriptomic landscapes. Nat. Commun. 7, 11479 (2016).

35. Cancer Genome Atlas Research, N.. Integrated genomic analyses of ovarian carcinoma. Nature 474, 609-615 (2011).

36. Li, H. \& Durbin, R. Fast and accurate short read alignment with BurrowsWheeler transform. Bioinformatics 25, 1754-1760 (2009).

37. Cibulskis, K. et al. Sensitive detection of somatic point mutations in impure and heterogeneous cancer samples. Nat. Biotechnol. 31, 213-219 (2013).

38. Wang, K., Li, M. \& Hakonarson, H. ANNOVAR: functional annotation of genetic variants from high-throughput sequencing data. Nucleic Acids Res. 38, e164 (2010).

\section{Acknowledgements}

We are grateful to the Genetically Engineered Mouse Facility (GEMF) and the Sequencing and Microarray Facility (SMF) at MD Anderson Cancer Center (supported by CA16672). We also acknowledge Drs. Vidya Sinha and Ramon E. Flores Gonzalez for teaching us mouse mammary intraductal injections, and Drs. Amanda Wasylishen and Denada Dibra for critical reading of the manuscript. This work is supported by MD Anderson Odyssey Program-Theodore N. Law Endowment for Scientific Achievement (Y.Z.), MD Anderson Institution Research Grant (S.X.), and NIH grant CA82577 (G.L.)

\section{Author contributions}

G.L. conceived the initial concept, developed design approach, interpreted the data and revised and finalized the manuscript. Y.Z. and S.X. contributed equally to this study by planning and performing most of the experiments, discussing and interpreting the data, and drafting the manuscript. B.L. proposed tumor heterogeneity and evolution study, performed bioinformatics analysis and assisted with manuscript writing. V.P. assisted with southern blot assays, discussion and critical reading of the manuscript. F.C. helped with characterizing the $\operatorname{Tr} p 53^{\mathrm{wm}-R 172 \mathrm{H}}$ allele. G.C., A.C.E., and P.Y. provided technical assistance. M.J.Y. and A.K.E. performed pathological diagnosis of mouse tissues. N.E.N. assisted the whole exome sequencing data analysis and interpretation.

\section{Additional information}

Supplementary Information accompanies this paper at https://doi.org/10.1038/s41467018-06146-9.

Competing interests: The authors declare no competing interests.

Reprints and permission information is available online at http://npg.nature.com/ reprintsandpermissions/

Publisher's note: Springer Nature remains neutral with regard to jurisdictional claims in published maps and institutional affiliations.

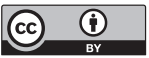

Open Access This article is licensed under a Creative Commons Attribution 4.0 International License, which permits use, sharing, adaptation, distribution and reproduction in any medium or format, as long as you give appropriate credit to the original author(s) and the source, provide a link to the Creative Commons license, and indicate if changes were made. The images or other third party material in this article are included in the article's Creative Commons license, unless indicated otherwise in a credit line to the material. If material is not included in the article's Creative Commons license and your intended use is not permitted by statutory regulation or exceeds the permitted use, you will need to obtain permission directly from the copyright holder. To view a copy of this license, visit http://creativecommons.org/ licenses/by/4.0/.

(c) The Author(s) 2018 